Conclusions Optimization of the deployment of BCMA in the Geriatric units of Toulouse CHU allows us to plan the development of this practise over a large number of clinical departments at a later date.

No conflict of interest.

\section{PHC-008 DEVELOPMENT AND APPLICATION OF A SIMPLE LC-MS METHOD FOR THE DETERMINATION OF PLASMA RILPIVIRINE CONCENTRATIONS}

doi:10.1136/ejhpharm-2013-000276.353

'M Shibata, 'M Takahashi, 'N Fukushima, 'F Yamaguchi, 'T Nomura, ${ }^{2} Y$ Yokomaku, ${ }^{2}$ W Sugiura. 'National Hospital Organization Nagoya Medical Center, Pharmacy, Nagoya, Japan; ${ }^{2}$ National Hospital Organization Nagoya Medical Center, Clinical Research Center, Nagoya, Japan

Background Rilpivirine is a second-generation non-nucleoside reverse transcriptase inhibitor that is highly potent against both wild-type and drug-resistant HIV-1 strains. The quantification of rilpivirine in human plasma is important to support clinical studies.

Purpose Rilpivirine was just approved in May 2012 in Japan. Therefore, pharmacokinetic studies of rilpivirine have still not been completed in Japanese patients. We intended to develop a conventional method for determining plasma rilpivirine concentrations and compare plasma rilpivirine concentrations of Japanese HIV-1 infected patients with those of foreign healthy volunteers.

Materials and Methods We used a Waters Alliance 2695 HPLC and a Micromass ZQ-2000 MS, controlled with MassLynx version 4.0 software. Our method involves rapid liquid-liquid drug extraction from plasma and use of gradient elution on a reversed-phase C18 column. We recruited 34 Japanese HIV-1 infected patients who were treated with a rilpivirine-containing regimen at the National Hospital Organization Nagoya Medical Center, Japan. All patients had been given $75 \mathrm{mg}$ rilpivirine once daily in combination with other antiretrovirals.

Results The LC-MS method established was validated by estimating the precision and accuracy for inter- and intraday analysis in the concentration range of $18-715 \mathrm{ng} / \mathrm{ml}$. The calibration curve was linear in this range. Average accuracy ranged from 100.0 to $100.6 \%$. Relative standard deviations of both inter- and intraday assays were less than $3.3 \%$. In this study, mean rilpivirine plasma concentration for Japanese patients at trough was $58 \mathrm{ng} / \mathrm{ml}(\mathrm{n}=18)$. Mean rilpivirine concentration at peak was $126 \mathrm{ng} / \mathrm{ml}(\mathrm{n}=6)$. These levels were higher than rilpivirine concentrations seen in trials with healthy foreign volunteers.

Conclusions Our LC-MS method provides a conventional, accurate and precise way of determining rilpivirine in human plasma. In clinical practise, AUC of rilpivirine for Japanese HIV-1 infected patients is larger in comparison with foreign data. We think that this was caused by the poor build of Japanese HIV-1 infected patients.

No conflict of interest.

\section{PHC-009 DRUG DOSE ADJUSTMENT IN RENAL FAILURE}

doi:10.1136/ejhpharm-2013-000276.354

AM Rizo Cerdá, P Selvi-Sabater, MC Sanchez-Mulero, A Boso-Ribelles, B Arribas-Diaz, A Morego-Soler, N Manresa-Ramon, I Sanchez-Martinez, MD Najera-Perez, I SanchezQuilez. Hospital Morales Meseguer, Pharmacy, Murcia, Spain

Background In renal failure, alteration in the pharmacokinetics increases the frequency of overdoses.

Purpose To evaluate pharmaceutical care using a computer programme for drug dose adjustment in renal failure.
Materials and Methods The study period lasted from September 2011 to January 2012 (inclusive), in a 420-bed hospital. Every day creatinine values over $130 \mathrm{mmol} / \mathrm{l}$ were filtered. Treatment was reviewed and we obtained creatinine clearance values (Crockcoft \& Gault) of selected patients. After consulting the drug dose adjustment on the sheet and in Micromedex, a report was sent with the pharmaceutical recommendation.

Results There were 68 interventions for the 2147 patients studied: Internal Medicine (34) Cardiology (1), Short Stay Unit (5), Orthopaedics (7), Urology (5), Haematology (7) Surgery (5), Neurology (1), Intensive Care Unit (ICU) (2) Oncology (1). 55.9\% of notifications were for changes in the dose of enoxaparin (38), $11.8 \%$ of amoxicillin-clavulanic acid (8), piperacillin-tazobactam 14.7\% (10), $8.8 \%$ levofloxacin (6), 2.9\% meropenem (2), 2.9\% ciprofloxacin (2), $1.5 \%$ imipenem (1) and $1.5 \%$ aztreonam (1). The proportion of suggested changes accepted was $58.8 \%$ (40). $5.9 \%$ (4) discontinued treatment, $5.9 \%$ (4) were discharged and $29.4 \%$ (20) not changed. Of the latter, five were for changes in the pattern of enoxaparin in trauma patients, another 5 from Internal Medicine and 2 more from Haematology and ICU. The rest of them were changes in the pattern of antibiotics (imipenem 1, 2 levofloxacin, 1 meropenem, 1 ciprofloxacin, piperacillin-tazobactam 3) that were given out in the different services.

Conclusions A high percentage of doctors followed the recommendations. Part of the unaccepted tally corresponds to trauma patients whose prophylactic regimen of enoxaparin $(40 \mathrm{mg} / 24 \mathrm{~h}$ ) was not modified due to the service criteria. Some of the antibiotic prescriptions were not changed because of the severity of the patient's illness (1 levofloxacin and 1 Internal Medicine Meropenem Imipenem Oncology and 1). The rest were rejected without explanation.

No conflict of interest.

\section{PHC-010 DRUG INTERACTION: A CASE REPORT}

doi:10.1136/ejhpharm-2013-000276.355

E Fernández López, E Tevar Alfonso, MA Ocaña Gomez, JA De León Gil, I Plasencia García, J Merino Alonso, R Jurado López, E Marqués Guell. Hospital Nuestra Señora de Candelaria, Farmacia Hospitalaria, Santa Cruz de Tenerife, Spain

Background The serum concentration of valproic acid (VPA) in epilepsy patients is reduced to sub-therapeutic by the administration of carbapenems antibiotics.

Purpose Description of the interaction and communication to the Pharmacovigilance Center with yellow cards.

Materials and Methods A 66-year-old was admitted to the resuscitation unit after being operated on for perforation peritonitis secondary to cytomegalovirus. Treatment was with imipenem because the suspicion of extended-spectrum beta-lactamases (ESLB) organisms was confirmed. Concomitant treatment was with VPA $400 \mathrm{mg}-400 \mathrm{mg}-400 \mathrm{mg}$ due to an underlying disease, epilepsy. The pharmacy department was asked to cheque the VPA blood level: initially levels were within the therapeutic interval (TI), but at 24 hours after starting treatment with imipenem it decreased by $70 \%$ to below the TI. In addition, because of the proconvulsive properties of imipenem, the patient started to have convulsions.

After reporting the suspected interaction, the doctor decided to change the antibiotic to meropenem $1 \mathrm{~g} / 8 \mathrm{~h}$ and so eliminate at least the pharmacodynamic component of the interaction. After 24 hours of the change VPA levels continued to fall and at 48 hours were almost undetectable $(\leq 3 \mathrm{mcg} / \mathrm{mL})$. VPA dose was increased, 1000 mg-1200 mg-1000 mg, without the situation reversimg. After 30 days meropenem was suspended and VPA levels did not return to the TI until after approximately $120 \mathrm{~h}$.

Results Although the exact mechanism is unknown, it is suspected to be of the pharmacokinetic kind and at several levels: 